\title{
The microRNA cluster C19MC is deregulated in parathyroid tumours
}

\author{
Valentina Vaira, Francesca Elli ${ }^{1,2}$, Irene Forno ${ }^{2}$, Vito Guarnieri ${ }^{3}$, Chiara Verdelli ${ }^{4}$, \\ Stefano Ferrero ${ }^{5}$, Alfredo Scillitani ${ }^{6}$, Leonardo Vicentini ${ }^{7}$, Filomena Cetani $^{8}$, \\ Giovanna Mantovani ${ }^{1}$, Anna Spada ${ }^{1}$, Silvano Bosari ${ }^{2}$ and Sabrina Corbetta ${ }^{9}$ \\ Division of Pathology, Fondazione IRCCS Cà Granda Ospedale Maggiore Policlinico, Milan, Italy \\ ${ }^{1}$ Endocrinology Unit, Department of Clinical Sciences and Community, ${ }^{2}$ Department of Clinical/Surgical Pathophysiology and Organ Transplant, University of Milan and Division of \\ Pathology, Fondazione IRCCS Cà Granda Ospedale Maggiore Policlinico, Milan, Italy \\ ${ }^{3}$ Medical Genetics, IRCCS Hospital Casa Sollievo della Sofferenza, San Giovanni Rotondo, Foggia, Italy \\ ${ }^{4}$ Laboratory of Molecular Biology, IRCCS Policlinico San Donato, San Donato Milanese, Italy \\ ${ }^{5}$ Department of Biomedical, Surgical and Dental Sciences, University of Milan and Division of pathology, Fondazione IRCCS Ca' Granda Ospedale Maggiore Policlinico, Milan, Italy \\ ${ }^{6}$ Endocrinology Unit, IRCCS Hospital Casa Sollievo della Sofferenza, San Giovanni Rotondo, Foggia, Italy \\ ${ }^{7}$ Endocrine Surgery, Fondazione IRCCS Cà Granda Ospedale Maggiore Policlinico, Milan, Italy \\ ${ }^{8}$ Department of Endocrinology and Metabolism, University of Pisa, Pisa, Italy \\ ${ }^{9}$ Endocrinology and Diabetology Unit, Department of Biomedical Sciences for the Health, IRCCS Policlinico San Donato, University of Milan, Via Morandi 30,20097 San Donato \\ Milanese, Italy \\ (Correspondence should be addressed to S Corbetta; Email: sabrina.corbetta@unimi.it)
}

\begin{abstract}
A subset of over-expressed microRNAs (miRNAs) identified in parathyroid carcinomas (Ca) compared to normal glands belongs to C19MC, a cluster on chromosome 19q13.4 involved in stem cell biology and tumourigenesis. In this study, the expression of C19MC-MIR371-3 clusters and the molecular mechanisms presiding their modulation were investigated in a series of six normal parathyroids, 24 adenomas (Ad), $15 \mathrm{Ca}$ and five matched metastases. The general expression levels of C19MC or MIR371-3 clusters in Ad lesions did not differ from normal glands, while they distinguished Ad from $\mathrm{Ca}$ at unsupervised hierarchical cluster analysis $(P=0.0008)$. MIR517C showed the most significant difference in expression between $\mathrm{Ca}$ and $\mathrm{Ad}(P=0.0003)$ and it positively correlated with serum calcium, parathormone and tumour weight. In regard to the molecular mechanism determining C19MC cluster activation, we could detect C19MC copy number (CN) gain in ten $\mathrm{Ca}(67 \%)$ extending distal to the MIR371-3 cluster in almost all samples. Conversely, only four Ad (16\%) showed C19MC amplification, with one case presenting distal genomic aberration to MIR371-3. Globally, CN variations of 19q13.4 loci were significantly associated with MIR517C up-regulation $(P=0.006)$. Opposite to normal glands where C19MC promoter was methylated, hypomethylation occurred in 15 out of 30 analysed tumours. Though the epigenetic status did not correlate with C19MC miRNA expression levels, loss of C19MC promoter methylation was significantly associated with $\mathrm{Ca}$ and metastatic disease $(P=0.01)$. In conclusion, $\mathrm{C} 19 \mathrm{MC}$ cluster aberrations are a characteristic of $\mathrm{Ca}$ with respect to Ad. Altogether, these evidences point towards a role for 19q13.4 miRNA clusters as oncogenes in parathyroid tumourigenesis.
\end{abstract}

Journal of Molecular Endocrinology (2012) 49, 115-124

\section{Introduction}

Primary hyperparathyroidism is a common endocrine disorder in post-menopausal women. Parathormone $(\mathrm{PTH})$ hypersecretion is sustained by a single parathyroid adenoma (Ad) in the majority of cases, while parathyroid carcinomas $(\mathrm{Ca})$ are rare $(<5 \%$ of cases) and invariably lethal. Though germ-line or somatic inactivating mutations of oncosuppressor genes (CDC73/HRPT2 and MENIN) frequently occur in parathyroid tumours, the molecular mechanisms of parathyroid tumourigenesis are still largely unknown.
MicroRNAs (miRNAs) are small, endogenously expressed non-protein coding RNAs $(\sim 19-23 \mathrm{nt}$ in length) that regulate gene expression. miRNAs interact with a huge number of target messenger RNAs and are recognised as key regulators of a broad range of cellular and developmental processes (Bushati \& Cohen 2007). Recent evidence indicates that alterations in miRNA genes play a critical role in cancer initiation and progression (Calin \& Croce 2007).

Recently, we produced data showing an altered miRNA expression pattern in parathyroid cancers compared with normal parathyroid tissue (Corbetta et al. 2010). Among the identified over-expressed 
miRNAs $(n=16)$, a subset $(50 \%)$ belonged to the primate-specific miRNA cluster C19MC mapping to chromosome 19q13.4. The $100 \mathrm{~kb}$ long C19MC cluster with 46 tandemly repeated miRNA genes encoding for 59 mature miRNAs is the largest cluster discovered so far in humans and accounts for about $8 \%$ of all known human miRNA genes (Bortolin-Cavaille et al. 2009). The large C19MC cluster shares a similar seed sequence with the closely distal MIR371-3 cluster, which is much smaller, spanning a region of $\sim 1050 \mathrm{bp}$ where four genes encode for seven mature miRNAs (Laurent et al. 2008). Reports recently described that members of the C19MC or MIR371-3 clusters are expressed by human embryonic stem cells (hESCs; Laurent $e t$ al. 2008, Li et al. 2009b, Ren et al. 2009) and over-expressed by human cancers (Toffanin et al. 2011). Activation of both clusters might be a general phenomenon in human tumourigenesis, as rearrangements of $19 \mathrm{q} 13.4$, genomic amplification and methylation abnormalities including the C19MC locus have been frequently found in several human neoplasias (Li et al. 2009a, Rippe et al. 2010, Toffanin et al. 2011). Finally, two members of the C19MC cluster, namely MIR517C and 520G, were shown to act as oncogenes, by promoting disease development in animal models ( $\mathrm{Li}$ et al. 2009a).

In this study, we identified a selective over-expression of a specific miRNA belonging to the C19MC cluster, namely MIR517C, in parathyroid Ca compared with benign Ad and normal tissues. Moreover, the expression pattern of C19MC and MIR371-3 cluster members was consistent with genomic amplification of $19 q 13.41$ in the majority of parathyroid Ca. Finally, C19MC promoter demethylation was associated with the presence of invasive tumour (parathyroid $\mathrm{Ca}$ ) and metastatic disease. These data point to a potential oncogenic role for C19MC miRNAs in parathyroid tumourigenesis.

\section{Materials and methods}

\section{Parathyroid tissue samples}

Paraffin-embedded samples from 15 parathyroid Ca and five matched metastasis, 24 parathyroid Ad and six normal parathyroid glands incidentally removed from normocalcemic patients having undergone thyroid surgery were collected. Specimens from parathyroid $\mathrm{Ca}$ and metastasis were sampled from the central portion of the tumours to avoid the contamination of parathyroid tissue with adjacent non-parathyroid tissue. Four parathyroid Ca have been previously described (Guarnieri et al. 2008, Corbetta et al. 2010). To avoid thyroid tissue contamination, normal

Table 1 Clinical and molecular features of the analysed parathyroid benign tumours

\section{C19MC CN ${ }^{1}$}

\begin{tabular}{|c|c|c|c|c|c|c|c|c|c|}
\hline Samples & Sex & Age (years) & $\mathrm{SCa}(\mathrm{mg} / \mathrm{dl})$ & PTH (pg/ml) & $\begin{array}{l}\text { Tumour } \\
\text { weight }(\mathrm{g})\end{array}$ & Cysts & Hs.7145628 & Hs. 7147457 & Hs. 4008868 \\
\hline Ad1 & $M$ & 65 & $11 \cdot 5$ & 235 & $1 \cdot 2$ & No & NA & NA & NA \\
\hline Ad3 & $\mathrm{F}$ & 53 & $11 \cdot 8$ & 151 & $2 \cdot 0$ & Yes & $1 \cdot 4$ & 0.6 & 0.9 \\
\hline $\mathrm{Ad} 4$ & $\mathrm{~F}$ & 53 & $11 \cdot 0$ & 336 & 3.5 & Yes & 0.7 & 0.2 & 0.9 \\
\hline Ad5 & $\mathrm{F}$ & 47 & $13 \cdot 4$ & 524 & $1 \cdot 8$ & Yes & 0.2 & $0 . \overline{5}$ & 0.4 \\
\hline Ad8 & $\mathrm{F}$ & 69 & $11 \cdot 1$ & 154 & 3.0 & No & 2.5 & $1 \cdot 1$ & $2 \cdot 6$ \\
\hline Ad9 & $M$ & 57 & $12 \cdot 8$ & 149 & $6 \cdot 0$ & No & 0.8 & 0.2 & 0.9 \\
\hline Ad10 & $\mathrm{M}$ & 57 & $11 \cdot 2$ & 100 & $5 \cdot 0$ & No & NA & NA & NA \\
\hline Ad11 & $\mathrm{F}$ & 53 & $11 \cdot 6$ & 137 & $4 \cdot 0$ & No & 0.7 & 0.4 & 0.6 \\
\hline Ad12 & $\mathrm{F}$ & 75 & $10 \cdot 6$ & 475 & 4.5 & No & 6.0 & 5.0 & $2 \cdot 5$ \\
\hline Ad13 & $M$ & 63 & $11 \cdot 6$ & 517 & $16 \cdot 0$ & No & 1.9 & 0.5 & $1 \cdot 3$ \\
\hline Ad14 & $\mathrm{F}$ & 42 & $11 \cdot 2$ & 304 & 3.8 & No & $2 \cdot 6$ & $1 \cdot 2$ & 2.0 \\
\hline Ad19 & $\mathrm{F}$ & 58 & $11 \cdot 4$ & 175 & 3.5 & No & NA & NA & NA \\
\hline $\mathrm{Ad} 20$ & $\mathrm{M}$ & 52 & $11 \cdot 1$ & 620 & 3.9 & No & $2 \cdot 5$ & $2 \cdot 9$ & $1 \cdot 7$ \\
\hline Ad21 & $\mathrm{M}$ & 45 & $13 \cdot 5$ & 1056 & $12 \cdot 0$ & No & $6 \cdot 3$ & 8.2 & $5 \cdot 7$ \\
\hline Ad22 & $\mathrm{M}$ & 86 & 11.9 & 542 & 13.0 & No & $4 \cdot 3$ & $2 \cdot 2$ & $2 \cdot 1$ \\
\hline Ad23 & $\mathrm{F}$ & 57 & $12 \cdot 0$ & 153 & 5.9 & No & 0.6 & 0.5 & 0.5 \\
\hline Ad24 & $M$ & 39 & $15 \cdot 4$ & 918 & $10 \cdot 0$ & No & $5 \cdot 2$ & $1 \cdot 3$ & 1.6 \\
\hline
\end{tabular}

F, female; M, male; SCa, serum calcium; PTH, parathormone; $\mathrm{CN}$, copy number; NA, not available.

${ }^{1}$ Gain in copy number at the indicated loci is indicated by bold text. 
parathyroid specimens were microdissected from paraffin-embedded sections of thyroid samples using a LMD6000 instrument (Leica Microsystems, Milan, Italy). Histologic classification of parathyroid $\mathrm{Ca}$ was established according to WHO-published guidelines (Bondenson et al. 2004). In all patients, fasting serum total calcium, phosphate and creatinine were measured by a multichannel autoanalyser. Intact PTH was determined by a chemiluminescent immunoassay (Nichols Advantage, Nichols Institute Diagnostics, San Clemente, CA, USA). Clinical data of parathyroid Ad are described in Table 1, whereas information about the parathyroid $\mathrm{Ca}$ specimens are shown in Table 2. The analysed secondarisms were brain metastasis from Ca10, lung metastasis from Ca11 and Ca13 and two distinct lymphatic metastases from Ca15 (Table 2). All carcinomatous and adenomatous specimens were from patients with primary hyperparathyroidism. Human term placenta samples were collected from healthy pregnant women $(n=3)$. The study protocol was approved by the Local Ethics Committee. Informed consent was obtained from all patients.

\section{DNA extraction and CDC73/HRPT2 gene mutations analysis}

Genomic DNA (gDNA) was extracted from the peripheral blood and the tumoural specimen of the patients with parathyroid Ca using standard techniques or a QIAamp DNA Mini kit (Qiagen) respectively according to the manufacturer's protocol. DNA samples were directly sequenced for $C D C 73 / H R P T 2$ gene mutations, as described previously (Cetani et al. 2004, Guarnieri et al. 2006). All gene mutations were confirmed on a second independent gDNA extraction.

\section{RNA extraction, reverse transcription and quantitative PCR}

Total RNA was isolated from paraffin-embedded tissues using MasterPure RNA purification kit (Epicentre Biotechnologies, Madison, WI, USA) according to the manufacturer's protocol. Following extraction, RNA was quantified spectrophotometrically and $500 \mathrm{ng}$ RNA from each sample were reverse transcribed in multiplex modality using the Megaplex Pool Primer sets A and B and the TaqMan MicroRNA Reverse Transcription kit (all from Applied Biosystems, Life Technologies). For single miRNA expression evaluation, $15 \mathrm{ng}$ cDNA of each sample were amplified with specific primers and probes for RNU48 and the target miRNAs (assay numbers are listed in Supplementary Table 1, see section on supplementary data given at the end of this article).

miRNAs expression relative to RNU48 was calculated using the comparative $C_{\mathrm{t}}$ method $\left(2^{-\Delta \Delta C_{\mathrm{t}}}\right)$ and the

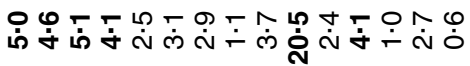

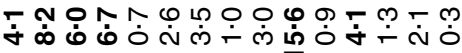

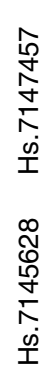

ஸ்

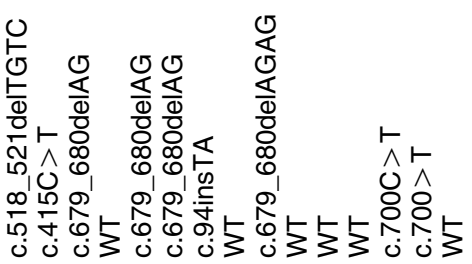

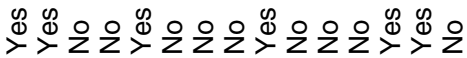

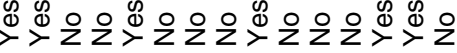

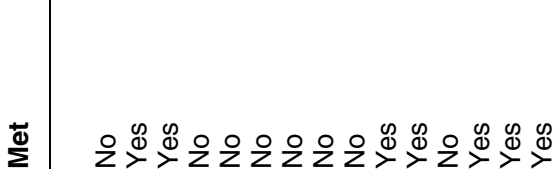

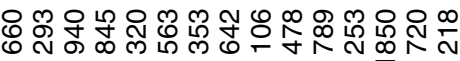

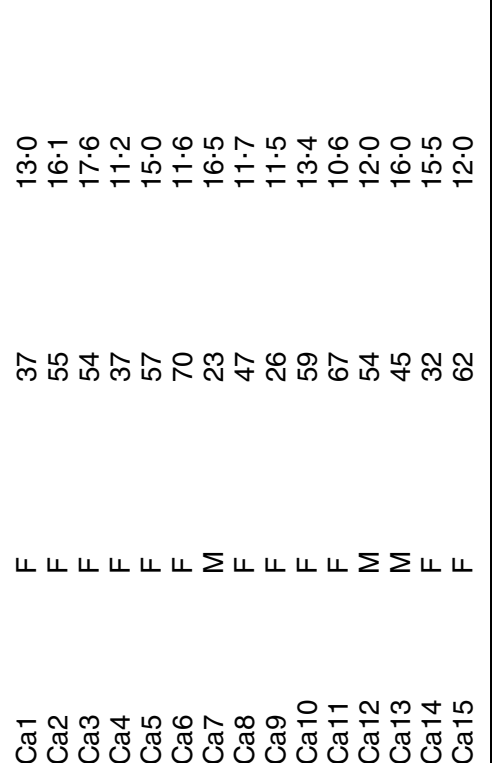


averaged value of normal parathyroids samples as calibrator $(1 \times$ sample $)$. As cut-off value for differential expression, we set a fold change (FC) of at least $2 \cdot 5$. When we compared sample groups (Ad, Ca or metastatic lesions), the FC was estimated on the median value of the miRNA in the category. All reagents and instruments were from Applied Biosystems.

\section{Copy number variation analysis}

TaqMan copy number (CN) assays were used to investigate genomic variation of C19MC and adjacent loci relative to the reference gene RNase $\mathrm{P}$. The assay identification numbers were Hs.7145628 (inside the C19 miRNA clusters, exact location 54170545), Hs.7147457 (inside the C19 miRNA clusters, exact location 54327727) and Hs4008868 (outside the C19 miRNA clusters, exact location 55225656). Genomic position of $\mathrm{CN}$ assays along the chr.19q13.41 locus is depicted in Fig. 1 according to the GRCh37/hg19 build from the USCS Genome database. The target and reference $\mathrm{CN}$ assays were run together in a duplex quantitative PCR and each sample was run in triplicate. For three Ad (Ad1, Ad11 and Ad19), gDNA was inadequate for $\mathrm{CN}$ analysis. Four normal parathyroid glands out of six were available for $\mathrm{CN}$ variation $(\mathrm{CNV})$ analysis. For CNV quantification in all specimens, instrument raw data were imported in CopyCaller v1.0 Software (Applied Biosystems, Life Technologies) and analysed without a calibrator sample. We set the

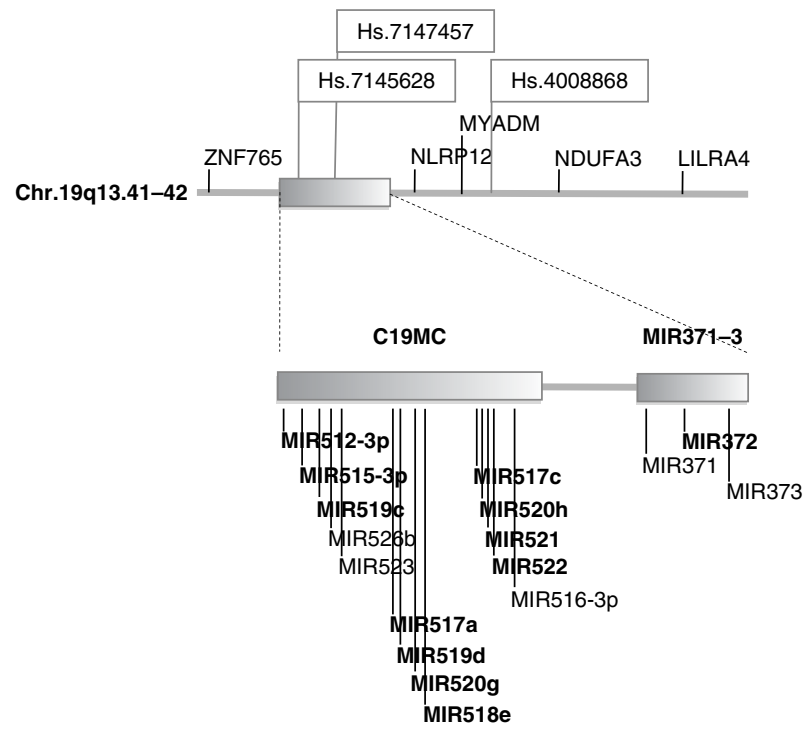

Figure 1 Diagram of genomic organisation of the chr.19q13.4142 locus. Relative positions of C19MC and MIR371-3 miRNA clusters, coding genes within the represented region boundaries are shown. Map positions are based on the hg19 build from the UCSC Genome Database (http://genome.ucsc.edu/). Dashed line denotes area enlargement. threshold for significant $\mathrm{CN}$ gain as at least four copies. All reagents, software and instruments were from Applied Biosystems.

\section{Methylation analysis}

The methylation status of the CpG island (CGI) localised $17 \cdot 6 \mathrm{~kb}$ upstream the C19MC was assessed by direct sequencing of PCR products amplified from bisulphite-treated gDNA (BiSeq Technique; Mantovani et al. 2010). Adequate DNA was available for six normal parathyroid glands, $14 \mathrm{Ad}$ and $12 \mathrm{Ca}$ and four metastases. The promoter region of C19MC was in silico analysed for the localisation of CGIs using ENSEMBL (http://www.ensembl.org/index.html), UCSC (http://genome.ucsc.edu/) and NCBI (http:// www.ncbi.nlm.nih.gov/) genome browsers. Primer sequences were specifically designed to amplify the modified DNA using MethPrimer (http://www.urogene. org/methprimer/index 1.html).

In particular, we analysed a $152 \mathrm{bp}$ fragment containing nine CpGs localised on chromosome 19 in position $54151441-54151592$. In brief, $360 \mathrm{ng}$ of each sample were treated with bisulphite by EZ-96 DNA Methylation-Gold Kit following the manufacturers' instructions and were PCR amplified using specific primer sets. PCR products were then purified and directly sequenced with the BigDye Terminator kit and 310 Genetic Analyzer (Perkin-Elmer Corp., Applied Biosystems). Then, the methylation status was analysed for the considered DNA locus with two software tools, which furthermore allow verifying bisulphite conversion efficiency: BiQ Analyzer (http://biq-analyzer.bioinf.mpi-inf.mpg.de/) and BISMA (http://biochem.jacobs-university.de/BDPC/ BISMA/). All samples were tested twice in order to confirm the reproducibility of the results.

\section{Statistical analysis}

Data for miRNA expression were presented as relative quantities data \pm s.D. (FCs over normal samples). Differences between normally distributed groups were investigated by univariate $t$ statistic or by Wilcoxon tests as indicated. Differences between frequencies were analysed by Fisher's exact test or $\chi^{2}$ test. miRNA expression levels as well as serum calcium and PTH were $\log 2$ transformed. Correlations were investigated by Spearman's rank test. For unsupervised hierarchical cluster analysis, miRNA expression data were mediannormalised and $\log 2$ transformed. Then data were imported in dChip Software (DNA Chip analyzer version 2010, http://biosun1.harvard.edu/complab/ dchip). A $P$ value $<0.05$ was considered as statistically significant. 


\section{Results}

\section{Expression of the C19MC and MIR371-3 miRNA clusters in parathyroid tumours}

In order to investigate the relevance of chr.19 miRNA clusters in parathyroid tumourigenesis, we first performed a gene expression survey of 15 and three miRNAs belonging, respectively, to the C19MC and the MIR371-3 clusters in a large series of parathyroid tumours with linked clinical-pathological annotation. Normal parathyroid samples were used to define baseline expression levels of the miRNAs and were arbitrarily set as $1 \times$. Looking at median expression values, none of the analysed miRNA was over-expressed by parathyroid Ad compared with controls (Supplementary Tables 2 and 3, see section on supplementary data given at the end of this article). Instead, seven or nine C19MC miRNAs and MIR372 respectively were over-expressed by $\mathrm{Ca}$ or metastatic lesions compared with normal parathyroid samples.

Globally, the expression profile of the analysed miRNAs clearly distinguished parathyroid Ad from $\mathrm{Ca}$ and metastatic disease $(P=0 \cdot 0008$; Fig. 2). All but one matched metastases (Met10) and primitive parathyroid Ca showed similar miRNA expression patterns (Fig. 2). Among all analysed miRNAs, MIR517C showed the most significant difference in expression between parathyroid Ad and Ca $(P=0 \cdot 0003$; Supplementary Table 2, see section on supplementary data given at the end of this article), and two Ad showed high MIR517C levels comparable with Ca (Ad23 and Ad24; Fig. 3). Elevated MIR517C levels ( $>2.5$ folds compared with normal glands) categorised carcinomatous samples with respect to $\operatorname{Ad}(P=0 \cdot 001$, Fisher's exact test $)$ and were significantly correlated with elevated expression of $12 \mathrm{C19MC}$ miRNAs or with MIR371/MIR372 (Fig. 2 and Table 3).

Considering clinical features of the parathyroid tumours, MIR520D-3p $(r=0 \cdot 318, P=0 \cdot 024)$, MIR520G $(r=0 \cdot 285, P=0 \cdot 039)$, MIR517C $(r=0 \cdot 302, P=0 \cdot 031)$ and MIR372 $(r=0.277, P=0.043)$ expression levels correlated positively with serum calcium levels. Similarly, MIR519D $(r=0 \cdot 328, P=0 \cdot 021)$, MIR520G $(r=0.385, P=0 \cdot 008)$, MIR518E $(r=0 \cdot 343, P=0 \cdot 016)$, MIR517C $(r=0.322, P=0.023)$ and MIR372 $(r=0.414$, $P=0 \cdot 004)$ expression levels correlated positively with serum PTH levels. Considering the subset of parathyroid Ad, MIR517C expression levels $(r=0 \cdot 404$, $P=0.025)$ correlated with tumour weights (Fig. 4).

The C19MC cluster has been documented to be abundant in human placenta. Therefore, we compared the expression levels of the selected miRNAs belonging to C19MC and MIR371-3 clusters in our series of parathyroid samples with three human term placentas.

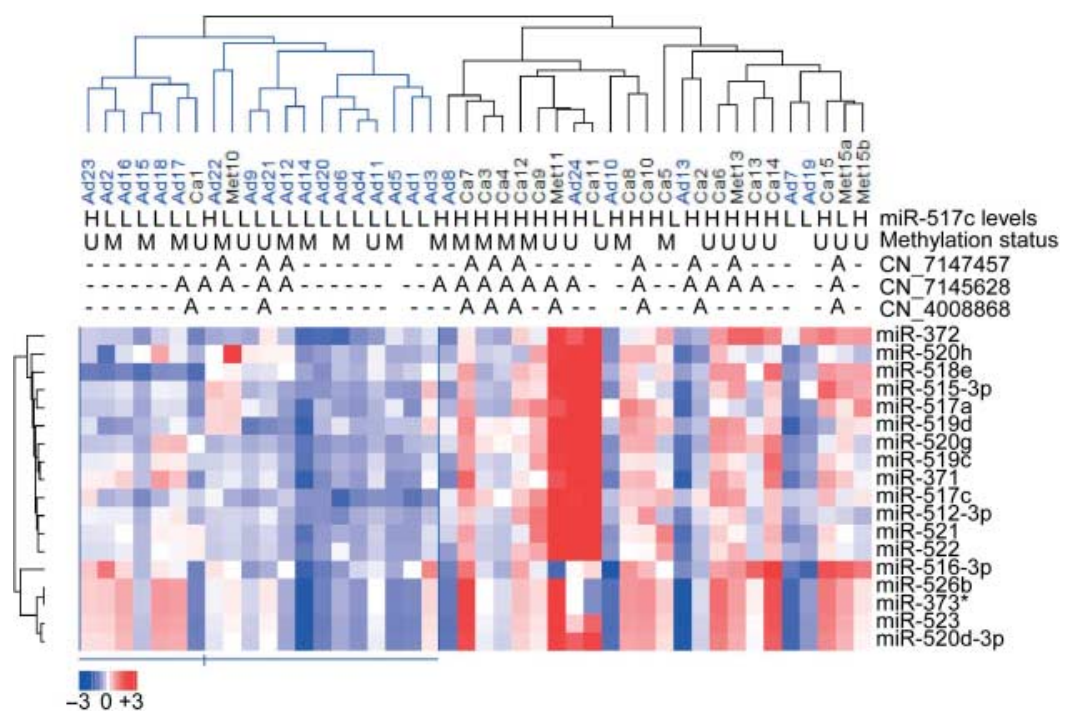

Figure 2 Chr.19 miRNA cluster expression profile categorises parathyroid tumours. Hierarchical cluster analysis clearly separates parathyroid carcinoma samples and corresponding metastases from adenomas according to the expression levels of C19MC and MIR371-3 miRNAs. Molecular (copy number gain) and epigenetic (C19MC promoter methylation) information of samples are indicated. MIR517C levels (high, $\mathrm{H}$ or low, L) were also used as molecular feature to categorise samples. The cluster branch enriched for parathyroid adenomas was also characterised for low MIR517C levels $(P=0.0008)$. Ad, parathyroid adenomas; Ca, carcinomas; Met, metastases; $\mathrm{U}$, hypomethylated C19MC promoter; M, methylated C19MC promoter; A, genomic amplification of the indicated locus. 


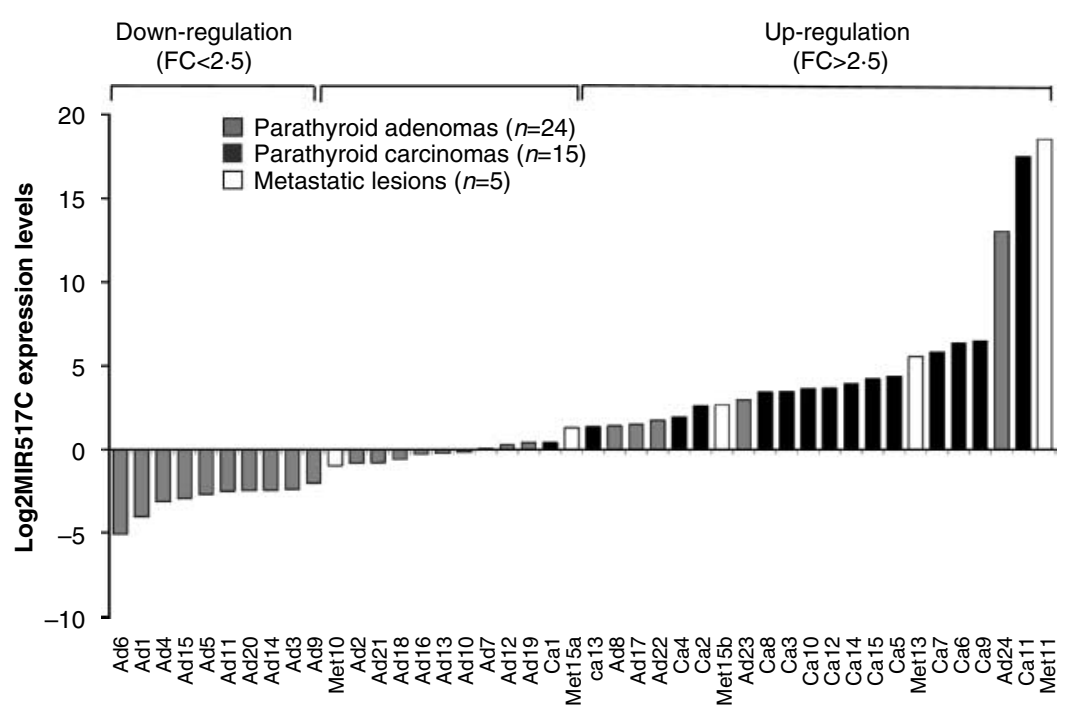

Figure 3 MIR517C expression levels in parathyroid adenomas (Ad), primitive carcinomas $(\mathrm{Ca})$ and matched metastatic lesions (Met). Data are expressed as fold increase (positive values) or decrease (negative values) on the averaged expression of the miRNA in normal parathyroid glands. Light grey bars, parathyroid adenomas; black bars, parathyroid carcinomas and white bars, matched metastatic lesions.

In agreement with the literature, we detected high expression of all investigated miRNAs except for MIR526B, MIR523, MIR516-3p, MIR371 and MIR373 (Supplementary Fig. 1, see section on supplementary data given at the end of this article). In regard to parathyroid $\mathrm{Ca}$, comparable median expression levels to human placentas could be documented for MIR519D, MIR518E, MIR517C and MIR372 (Supplementary Fig. 1, see section on supplementary data given at the end of this article).

\section{Genomic amplification of the chr.19q13.41 region is a frequent event in parathyroid tumours}

To discern mechanisms underpinning C19MC and MIR371-3 miRNA cluster over-expression in parathyroid $\mathrm{Ca}$, we investigated whether gain in allelic $\mathrm{CN}$ of the chr.19q13.41-42 region was involved. We could detect significant gain in CN either in miRNA cluster regions (Hs.7145628 and Hs.7147457 probes; Fig. 1) or at a distant position along chr.19q arm (Hs.4008868 probe; Fig. 1). Specifically, four out of 21 parathyroid Ad (19\%), ten out of $15 \mathrm{Ca}(67 \%)$ and four out of five matched metastases $(80 \%)$ showed amplification of the chr.19q13.41-42 locus for at least one probe $\left(P=0.004\right.$ by $\chi^{2}$ test $)$. The analysis of DNA CNV by the distal probe showed that the amplification extended through the distal MIR371-3 cluster in one Ad, six Ca and one metastatic lesion (Tables 1 and 2, Fig. 5). The increase in chr.19 $\mathrm{CN}$ in parathyroid $\mathrm{Ca}$ or matched metastases compared with Ad was statistically significant (Fig. 5; $P=0.003$ and $P=0.03$;
$P=0.015$ and $P=0.001$ for Hs.7145628, Hs. 7147457 and Hs.4008868 probes respectively).

As expression levels of MIR517C were representative of general C19MC miRNA expressions (Table 3), we investigated whether increased chr.19 $\mathrm{CN}$ in parathyroid samples were associated to elevated MIR517C expression ( $\mathrm{FC}>2 \cdot 5)$. A significant relationship could be established $(P=0 \cdot 006$, Fisher's exact test; Supplementary Fig. 1, see section on supplementary data given at the end of this article).

Table 3 MIR517C levels are a valuable indicator of global C19MC and MIR371-3 miRNA expression profiles. MIR517C levels categorised samples at hierarchical cluster analysis (Fig. 2). The absolute correlation was therefore calculated to reveal microRNAs associated by similarity in expression pattern

\begin{tabular}{|c|c|c|}
\hline microRNA & Correlation & $P$ value \\
\hline \multicolumn{3}{|c|}{ C19MC microRNA cluster } \\
\hline MIR519C & 0.96 & $7.98 \times 10^{-26}$ \\
\hline MIR521 & 0.96 & $3.44 \times 10^{-25}$ \\
\hline MIR520G & 0.95 & $1.66 \times 10^{-24}$ \\
\hline MIR512-3p & 0.94 & $1.92 \times 10^{-22}$ \\
\hline MIR515-3p & 0.94 & $3.14 \times 10^{-22}$ \\
\hline MIR522 & 0.93 & $2.19 \times 10^{-20}$ \\
\hline MIR519D & 0.92 & $9.47 \times 10^{-20}$ \\
\hline MIR517A & 0.92 & $7 \cdot 82 \times 10^{-19}$ \\
\hline MI518E & 0.90 & $3.50 \times 10^{-17}$ \\
\hline $\mathrm{MIR} 520 \mathrm{H}$ & 0.86 & $4.33 \times 10^{-14}$ \\
\hline MIR520D-3p & 0.84 & $4.74 \times 10^{-13}$ \\
\hline MIR523 & 0.73 & $1.01 \times 10^{-8}$ \\
\hline \multicolumn{3}{|c|}{ MIR371-3 microRNA cluster } \\
\hline MIR371 & 0.93 & $3.94 \times 10^{-20}$ \\
\hline MIR372 & 0.82 & $3.83 \times 10^{-12}$ \\
\hline
\end{tabular}




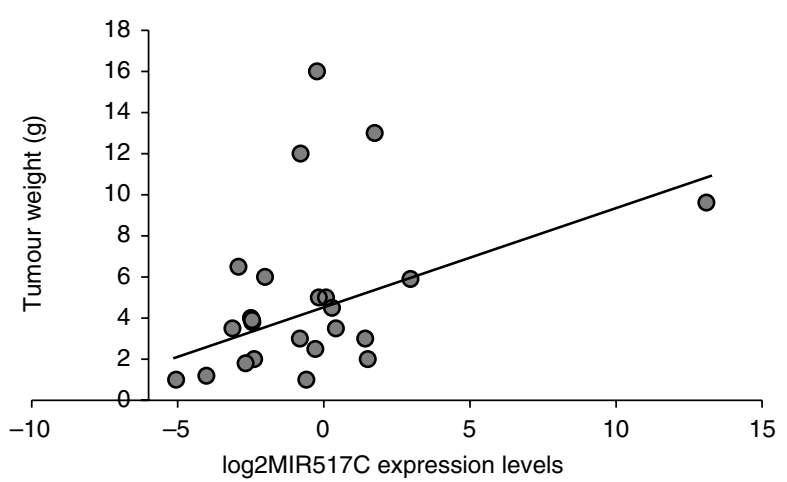

Figure 4 Correlations between MIR517C expression levels and clinical features. Elevated MIR517C levels significantly and positively correlated with tumour weight in parathyroid adenomas $(P=0.025)$. Filled circles, parathyroid Ad.

\section{C19MC promoter methylation status in parathyroid tumours}

DNA methylation of CpG-rich regions is a key event in epigenetic gene regulation or genomic imprinting. Therefore, we tested the hypothesis that a different epigenetic pattern in the C19MC promoter regulatory region might be related to deregulation of C19MC miRNA expression in parathyroid tumours. Compared with normal parathyroid glands (all methylated), hypomethylation of this CGI was detected in four parathyroid Ad (29\%), seven Ca $(58 \%)$ and four matched metastatic lesions $\left(100 \% ; P=0 \cdot 01, \chi^{2}\right.$ test). Gain in $\mathrm{CN}$ or hypomethylation was not mutually exclusive. Hypomethylation of the C19MC promoter region was not significantly associated with MIR517C expression levels in parathyroid samples $(P=0 \cdot 2$, Fisher's exact test $)$.

\section{Discussion}

In this study, we extended previous knowledge about chr.19q13.41-42 miRNA clusters in parathyroid tumourigenesis by analysing the expression of 15 and three miRNAs from C19MC or MIR371-3 clusters in a considerable series of parathyroid neoplastic lesions. This set of miRNAs was chosen according to their genomic location (UCSC Genome Database, Fig. 1) and their biological importance, as described previously (Toffanin et al. 2011, Augello et al. 2012). Furthermore, to get insight into deregulation of chr.19 miRNA clusters, allelic CNV of 19q13.41-42 locus (Rakheja et al. 2004, Li et al. 2009a, Rippe et al. 2010) and the epigenetic status of C19MC promoter (Tsai et al. 2009) were investigated. Importantly, our cohort comprised five matched metastatic lesions of four primitive parathyroid Ca. This sample set allowed us to analyse chr.19 molecular aberration in progressive disease.
The data showed that multiple molecular aberrations occurred in this locus and they are specific for parathyroid $\mathrm{Ca}$ or related metastatic lesions discriminating invasive disease from the less aggressive parathyroid tumours, i.e. Ad. In particular, MIR517C was identified as a potential surrogate marker to correctly classify samples in the appropriate diagnostic category. Indeed, MIR517C expression levels in parathyroid Ad were similar to those observed in normal parathyroid glands, whereas elevated MIR517C levels were detected in parathyroid $\mathrm{Ca}$ or matched metastases. C19MC and MIR371-3 miRNA clusters were concurrently expressed in parathyroid tumours, with MIR517C levels being significantly associated with MIR371 and MIR372 expression profiles.

MIR517C expression levels were also related to chr.19 copy gain. By contrast, the methylation status of C19MC promoter was not associated with the miRNA levels nor was it mutually exclusive with variation in allelic $\mathrm{CN}$. Nevertheless, our data showed progressive loss of methylation in C19MC promoter moving from parathyroid Ad to parathyroid $\mathrm{Ca}$ and metastatic lesions. A similar pattern of acquisition of molecular anomalies was evident from $\mathrm{CNV}$ analysis, where the amplification rate of chr.19 loci increased with disease severity. These observations point out that chr.19q13.41-42 region aberrations are an important hallmark of metastatic progression in parathyroid carcinogenesis.

miRNA deregulation has been recently demonstrated to be involved in cancer development. Different profiles of miRNA expression have been previously described in parathyroid $\mathrm{Ca}$ compared with normal parathyroid tissues (Corbetta et al. 2010, Rahbari et al. 2010), with members of C19MC miRNA being preliminarily

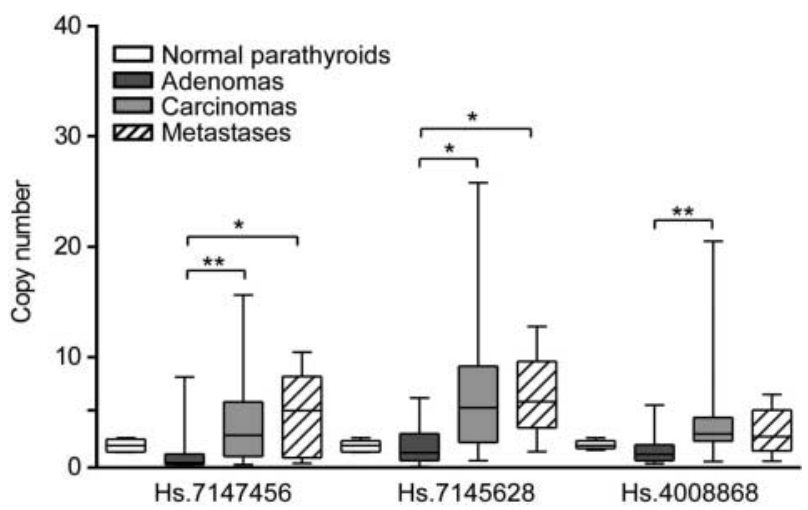

Figure 5 Chr.19q13.41-42 region is frequently amplified in parathyroid carcinomas or matched metastases. Copy number variation analysis was performed for three chr.19 genomic regions that spanned C19MC and MIR371-3 miRNA clusters (assay position is depicted in Fig. 1). Data are represented as box-whisker diagrams. White boxes represent normal parathyroid samples; black boxes, adenomas; grey boxes, parathyroid carcinomas and dashed boxes, metastases. ${ }^{\star} P<0.05 ;{ }^{\star \star} P<0.01$. 
identified as up-regulated in parathyroid Ca compared with normal parathyroid glands (Corbetta et al. 2010).

The C19MC cluster is considered a paternally imprinted 'embryonic' miRNAs cluster, which is expressed in hESCs and rapidly down-regulated during the differentiation process (Lin et al. 2010). Consistent with the notion that oncogenic miRNAs are highly expressed in ESCs, C19MC miRNAs are silenced in the majority of normal cells (Tsai et al. 2009) and overexpressed in a number of human neoplasias. Indeed, C19MC has been found to be abundantly expressed in term placenta (Liang et al. 2007).

In accordance with the literature, the analysed term placentas displayed high expression levels of all investigated C19MC miRNAs but MIR526B, MIR523 and MIR516-3p. This expression pattern was similar to those detected in parathyroid Ca though differences in over-expression intensities could be documented (Supplementary Fig. 1, see section on supplementary data given at the end of this article).

In the present series, one parathyroid Ad (Ad24, Table 1) showed high chr.19 miRNAs similar to Ca or term placenta samples and three more Ad showed CN gain in at least one analysed loci (Table 1). These Ad were clinically characterised by severe PTH hypersecretion and high tumour weight. Moreover, considering parathyroid adenomatous lesions per se, we found that higher MIR517C expression levels were positively correlated with increased tumour weight.

Similar to what was reported for the C19MC cluster, the MIR371-3 cluster has been implicated in malignant growth (Voorhoeve et al. 2006, Cho et al. 2009, Wang et al. 2010). Interestingly, the MIR371-3 cluster has also been involved in stem cell maintenance or differentiation and its expression has been reported to be specific for hESCs (Suh et al. 2004). In the present series, MIR371 and MIR372 were significantly upregulated in parathyroid $\mathrm{Ca}$ and matched metastases. The observations that both chr.19 miRNA clusters play a role in stem cell maintenance ( $\mathrm{Li}$ et al. 2009a), together with the identification in parathyroid tumours of a subpopulation with progenitor features (Corbetta et al. 2009), prompt us to speculate that deregulation of these miRNAs may contribute to maintenance of a tumour-associated parathyroid progenitor cell niche.

As previously described for subsets of aggressive neuroectodermal brain tumours (Li et al. 2009a), or hepatocellular Ca (Toffanin et al. 2011, Augello et al. 2012), our results showed that parathyroid Ca also displayed genomic or epigenetic abnormalities in the chr.19q13.41-42 region compared with normal or adenomatous samples. The analysis of $\mathrm{CNV}$ at the C19MC locus identified an amplification of this region in two-thirds of parathyroid Ca extending distal to the MIR371-3 cluster in most of the amplified samples. These results are in agreement with the data showing
DNA CN alterations by CGH in parathyroid neoplasia, with gains at chromosome $19 q$ in $20 \%$ of $\mathrm{Ca}$ and about $5 \%$ of Ad (Kytola et al. 2000).

The methylation status of promoter regions has also been analysed in parathyroid tumours (Starker et al. 2011) and frequent hypermethylation of RIZ1/PRDM2, $C D K N 2 A, R A S S F 1 A$ and APC genes has been reported (Carling et al. 2003, Juhlin et al. 2010, Svedlund et al. 2010).

The C19MC cluster is silenced in most cells by hypermethylation of a distal CpG-rich region localised $17 \cdot 6 \mathrm{~kb}$ upstream of the C19MC (Tsai et al. 2009). Our analysis of the methylation status in parathyroid tumours showed that the C19MC promoter region was hypomethylated in 29, 58 or $100 \%$ of $\mathrm{Ad}$, Ca or metastatic samples, respectively, therefore being a marker of aggressive parathyroid tumours. Nonetheless, hypomethylation and $\mathrm{CN}$ amplification of the C19MC cluster were not mutually exclusive. Admittedly, $\mathrm{CN}$ amplification and hypomethylation were not unequivocally associated with MIR517C up-regulation, discrepant data being observed in a small number of tumours.

In conclusion, this study showed that C19MC and MIR371-3 miRNA clusters were reactivated in a consistent proportion of parathyroid Ca possibly due to genomic gains of chr.19 loci. The data here presented pointed towards an oncogenic role of MIR517C in parathyroid tumourigenesis and deregulation of this cluster might contribute to survival and growth of cell populations with stem/progenitor function. Importantly, we evidenced that molecular aberration in chr.19q13.41-42 region is a marker of aggressive tumour in parathyroid neoplasia that could be helpful in its diagnostic management. Further understanding of function and regulation of these miRNAs and their target genes might provide the basis for identifying novel therapeutic approaches.

\section{Supplementary data}

This is linked to the online version of the paper at http://dx.doi.org/ 10.1530/JME-11-0189.

\section{Declaration of interest}

The authors declare that there is no conflict of interest that could be perceived as prejudicing the impartiality of the research reported.

\section{Funding}

The study was supported in part by grants to S B from the Fondazione Berlucchi, Fondazione Cariplo and the Ministero della Salute ' $5 \times 1000$ ' and to $\mathrm{S}$ C by IRCCS Policlinico San Donato research found. 


\section{Author contribution statement}

$\mathrm{V} \mathrm{V}$ and $\mathrm{S} \mathrm{C}$ were responsible of the study and wrote the manuscript; F E, I F, V G and C V performed molecular experiments; A S, L V, F C and $\mathrm{S} C$ enrolled patients and collected clinical and hormonal data as well as biological specimens; S F performed histological analysis; G M supervised methylation analysis; A S and S B supervised the study and critically reviewed the manuscript.

\section{References}

Augello C, Vaira V, Caruso L, Destro A, Maggioni M, Park YN, Montorsi M, Santambrogio R, Roncalli M \& Bosari S 2012 MicroRNA profiling of hepatocarcinogenesis identifies C19MC cluster as a novel prognostic biomarker in hepatocellular carcinoma. Liver International 32 772-882. (doi:10.1111/j.1478-3231.2012.02795.x)

Bondenson L, Grimelius L, DeLellis RA, Lloyd R, Akerstrom G, Larsson C, Arnold A, Eng C, Shane E \& Bilezikian JP 2004 Parathyroid carcinoma. In Pathology and Genetics. Tumors of Endocrine Organs. WHO Classification of Tumors, pp 128-132. Eds RA DeLellis, RV Lloyd, PU Heitz \& C Eng. Lyon: IARC Press.

Bortolin-Cavaille ML, Dance M, Weber M \& Cavaille J 2009 C19MC microRNAs are processed from introns of large Pol-II, nonprotein-coding transcripts. Nucleic Acids Research 37 3464-3473. (doi:10.1093/nar/gkp205)

Bushati N \& Cohen SM 2007 microRNA functions. Annual Review of Cell and Developmental Biology 23 175-205. (doi:10.1146/annurev. cellbio.23.090506.123406)

Calin GA \& Croce CM 2007 Chromosomal rearrangements and microRNAs: a new cancer link with clinical implications. Journal of Clinical Investigation 117 2059-2066. (doi:10.1172/JCI32577)

Carling T, Du Y, Fang W, Corres P \& Huang S 2003 Intragenic allelic loss and promoter hypermethylation of the RIZ1 tumor suppressor gene in parathyroid tumors and pheochromocytomas. Surgery 134 932-939. (doi:10.1016/S0039-6060(03)00422-7)

Cetani F, Pardi E, Borsari S, Viacava P, Dipollina G, Cinferotti L, Ambrogini E, Gazzero E, Colussi G, Berti P et al. 2004 Genetic analyses of the HRPT2 gene in primary hyperparathyroidism: germline and somatic mutations in familial and sporadic parathyroid tumors. Journal of Clinical Endocrinology and Metabolism 89 5583-5591. (doi:10.1210/jc.2004-0294)

Cho WJ, Shin JM, Kim JS, Lee MR, Hong KS, Lee JH, Koo KH, ParkJW \& Kim KS 2009 miR-372 regulates cell cycle and apoptosis of ags human gastric cancer cell line through direct regulation of LATS2. Molecular Cell 28 521-527. (doi:10.1007/s10059-009-0158-0)

Corbetta S, Belicchi M, Pisati F, Meregalli M, Eller-Vainicher C, Vicentini L, Beck-Peccoz P, Spada A \& Torrente Y 2009 Expression of parathyroid-specific genes in vascular endothelial progenitors of normal and tumoral parathyroid glands. American Journal of Pathology 175 1200-1207. (doi:10.2353/ajpath.2009.080979)

Corbetta S, Vaira V, Guarnieri V, Scillitani A, Eller-Vainicher C, Ferrero S, Vicentini L, Chiodini I, Bisceglia M, Beck-Peccoz P et al. 2010 Differential expression of microRNAs in human parathyroid carcinomas compared with normal parathyroid tissue. EndocrineRelated Cancer 17 135-146. (doi:10.1677/ERC-09-0134)

Guarnieri V, Scillitani A, Muscarella LA, Battista C, Bonfitto N, Bisceglia M, Minisola S, Mascia ML, D'Agruma L \& Cole DEC 2006 Diagnosis of parathyroid tumors in familial isolated hyperparathyroidism with HRPT2 mutation: implications for cancer surveillance. Journal of Clinical Endocrinology and Metabolism 91 2827-2832. (doi:10.1210/jc.2005-1239)

Guarnieri V, Bisceglia M, Bonfitto N, Cetani F, Marcocci C, Minisola S, Battista C, Cole DE \& Scillitani A 2008 Re: familial hyperparathyroidism: surgical outcome after 30 years of follow-up in three families with germline HRPT2 mutations. Surgery 144 839-840. (doi:10.1016/j.surg.2008.08.008)
Juhlin CC, Kiss NB, Villablanca A, Haglund F, Nordenstrom J, Hoog A \& Larsson C 2010 Frequent promoter hypermethylation of the APC and RASSF1A tumour suppressors in parathyroid tumours. PLOS ONE 5 e9472. (doi:10.1371/journal.pone.0009472)

Kytola S, Farnebo F, Obara T, Isola J, Grimelius L, Farnebo L-O, Sandelin K \& Larsson C 2000 Patterns of chromosomal imbalances in parathyroid carcinomas. American Journal of Pathology 157 579-586. (doi:10.1016/S0002-9440(10)64568-3)

Laurent LC, Chen J, Ulitsky I, Mueller FJ, Lu C, Shamir R, Fan JB \& Loring JF 2008 Comprehensive microRNA profiling reveals a unique human embryonic stem cell signature dominated by a single seed sequence. Stem Cells 26 1506-1516. (doi:10.1634/stemcells. 2007-1081)

Li M, Lee KF, Lu Y, Clarke I, Shih D, Eberhart C, Collins VP, Van Meter T, Picard D, Zhou L et al. 2009a Frequent amplification of a chr19q13.41 microRNA polycistron in aggressive primitive neuroectodermal brain tumors. Cancer Cell 16 533-546. (doi:10.1016/j.ccr. 2009.10.025)

Li SS, Yu SL, Kao LP, Tsai ZY, Singh S, Chen BZ, Ho BC, Liu YH \& Yang PC 2009b Target identification of microRNAs expressed highly in human embryonic stem cells. Journal of Cellular Biochemistry 106 1020-1030. (doi:10.1002/jcb.22084)

Liang Y, Ridzon D, Wong L \& Chen C 2007 Characterization of microRNA expression profiles in normal human tissues. $B M C$ Genomics 8 166. (doi:10.1186/1471-2164-8-166)

Lin S, Cheung WKC, Chen S, Lu G, Wang Z, Xie D, Li K, Lin MCM \& Kung H 2010 Computational identification and characterization of primate-specific microRNAs in human genome. Computational Biology and Chemistry 34 232-241. (doi:10.1016/j.compbiolchem. 2010.08.001)

Mantovani G, De Sanctis L, Barbieri AM, Elli FM, Bollati V, Vaira V, Labarile P, Bondioni S, Peverelli E, Lania AG et al. 2010 Pseudohypoparathyroidism and GNAS epigenetic defects: clinical evaluation of Albright hereditary osteodystrophy and molecular analysis in 40 patients. Journal of Clinical Endocrinology and Metabolism 95 651-658. (doi:10.1210/jc.2009-0176)

Rahbari R, Holloway AK, Mei He, Khanafshar E, Clark O \& Kebebew E 2010 Identification of differentially expressed microRNA in parathyroid tumors. Annals of Surgical Oncology 18 1158-1165. (doi:10.1245/s10434-010-1359-7)

Rakheja D, Margraf LR, Tomlinson GE \& Schneider NR 2004 Hepatic mesenchymal hamartoma with translocation involving chromosome band 19q13.4: a recurrent abnormality. Cancer Genetics and Cytogenetics 153 60-63. (doi:10.1016/j.cancergencyto.2003.12.004)

Ren J, Jin P, Wang E, Marincola FM \& Stroncek DF 2009 MicroRNA and gene expression patterns in the differentiation of human embryonic stem cells. Journal of Translational Medicine 720. (doi:10.1186/1479-5876-7-20)

Rippe V, Dittberner L, Lorenz VN, Driescner N, Nimzyk R, Sendt W, Junker K, Belge G \& Bullerdiek J 2010 The two stem cell microRNA gene clusters C19MC and miR-371-3 are activated by specific chromosomal rearrangements in a subgroup of thyroid adenomas. PLOS ONE 5 e9485. (doi:10.1371/journal.pone.0009485)

Starker LF, Svedlund J, Udelsman R, Dralle H, Akerstrom G, Westin G, Lifton RP, Bjorklund P \& Carling T 2011 The DNA methyloma of benign and malignant parathyroid tumors. Genes, Chromosomes $\mathcal{E}$ Cancer 50 735-745. (doi:10.1002/gcc.20895)

Suh M-R, Lee Y, Kim JY, Kim S-K, Moon S-H, Lee JY, Cha K-Y, Chung HM, Yoon HS, Moon SY et al. 2004 Human embryonic stem cells express a unique set of microRNAs. Developmental Biology 270 488-498. (doi:10.1016/j.ydbio.2004.02.019)

Svedlund J, Auren M, Sundstrom M, Dralle H, Akerstrom G, Bjorklund P \& Westin G 2010 Aberrant WNT/ $\beta$-catenin signalling in parathyroid carcinoma. Molecular Cancer 9 294. (doi:10.1186/ 1476-4598-9-294)

Toffanin S, Alsinet C, Cornella H, Sia D \& Llovet JM 2011 microRNAs and the MYC network: a major piece in the puzzle of liver cancer. Gastroenterology 140 2138-2140. (doi:10.1053/j.gastro.2011.04.023) 
Tsai KW, Kao HW, Chen HC, Chen SJ \& Lin WC 2009 Epigenetic control of the expression of a primate-specific microRNA cluster in human cancer cells. Epigenetics 4 587-592. (doi:10.4161/epi.4.8. 10230)

Voorhoeve PM, le Sage C, Schrier M, Gillis AJ, Stoop H, Nagel R, Liu YP, van Duijse J, Drost J, Griekspoor A et al. 2006 A genetic screen implicates miRNA-372 and miRNA-373 as oncogenes in testicular germ cell tumors. Cell 124 1169-1181. (doi:10.1016/ j.cell.2006.02.037)
Wang J, Liu X, Wu H, Ni P, Gu Z, Qiao Y, Chen N, Sun F \& Fan Q 2010 CREB up-regulates long non-coding RNA, HULC expression through interaction with microRNA-372 in liver cancer. Nucleic Acids Research 38 5366-5383. (doi:10.1093/nar/gkq285)

Received in final form 1 July 2012

Accepted 5 July 2012

Made available online as an Accepted Preprint 5 July 2012 